\title{
STEADY RADIATIVE FREE CONVECTIVE FLOW ALONG A Vertical Flat Plate in the Presence of Magnetic FIELD
}

\author{
Md. Enamul Karim ${ }^{1}$ and M. J. Uddin ${ }^{1}$ \\ ${ }^{1}$ Faculty of Science \& Information Technology, Daffodil International University. \\ E-mail address: ekarim_du@yahoo.com,jas_fluidm@yahoo.com
}

\begin{abstract}
Steady two-dimensional Magneto hydrodynamic free convection flow with thermal radiation in the presence of magnetic field along a vertical flat plate is concerned in the present study. The fluid is taken to be gray, absorbing-emitting radiation. The non-linear governing equations have been transformed by the usual similarity transformation to a system of ordinary differential equations. These dimensionless similar equations are then solved numerically employing the Nachtsheim-Swigert shooting iteration technique along with sixth order Runge-Kutta integration scheme. Finally the effects of the pertinent parameters are examined.
\end{abstract}

Keywords: MHD flow, radiation, magnetic field, kinematic viscosity and thermal diffusivity.

\section{Introduction}

Considerable interest has recently been shown in radiation interaction with free convection for heat transfer in fluid. This is due to the significant role of thermal radiation in the surface heat transfer when convection heat transfer is small particularly in free convection problems involving absorbing-emitting fluids.

The free convection processes involving the combined mechanism of heat and mass transfer are encountered in many natural processes, in many industrial applications and in many chemical processing systems.

In recent years, the study of free convective mass transfer flow has become the object of extensive research as the effects of heat transfer along with mass transfer effects are dominant features in many engineering applications such as rocket nozzles, cooling of nuclear reactors, high sinks in turbine blades, high speed aircrafts and their atmospheric re-entry, chemical devices and process equipments.

One of the initiators of this problem of radiation transfer in a vertical surface is Goody

Date of submission : 24.08.2010 Date of acceptance : 16.05.2011
(1956), who considered a neutral fluid. Cess (1966), on the other hand, considered an absorbing-emitting gray fluid with a black vertical plate. His solution was based on perturbation technique and was applicable for small values of the conduction- radiation interaction parameter. Novotny et al. (1974), however, made a non-gray analysis employing the method of local non-similarity and the continuous correlation of Tien and Lowder (1966) to account for the absorption. The effects of radiation on free convection flow of a gas past a semi infinite flat plate was studied by Soundalgekar and Takhar (1981) using the Cogley-Vincentine-Giles equilibrium model. Ali et al. (1984) studied the same effects on natural convection flow but over a vertical surface in a gray gas. Following Ali et al., Mansour (1990) studied the interaction of mixed convection with thermal radiation in laminar boundary layer flow over a horizontal, continuous moving sheet with suction and injection. At the same time Alabraba et al. (1992) studied the same problem of free convection interaction with thermal radiation in a hydro-magnetic boundary layer taking into account the binary chemical reaction and less attended Soret-Doufour effects.

By using the Rosseland diffusion approximation (1976), a study of the combined unsteady free convective dynamic boundary layer and thermal radiation boundary layer at a semi-infinite vertical plate was made by Sattar and Kalim (1996). Hossain and Takhar (1996) also analyzed the same effect of radiation using the Rosseland approximation in a mixed convection flow of an optically dense viscous incompressible

fluid past a heated vertical plate with a free uniform stream velocity and surface temperature. Since suction is the best control 
method of boundary layer growth in the presence of radiation El-Arabawy (2003) studied the effect of suction/injection on a micropolar fluid past a continuously moving plate. Ferdows et al. (2004), however, considered a variable suction in a boundary layer flow at a vertical plate with thermal radiation interaction with convection. Very recently Samad and Rahman (2006) investigated the thermal radiation interaction on an absorbing emitting fluid past a vertical porous plate immersed in a porous medium. They (2006) considered an unsteady MHD flow and observed that magnetic field can control the heat transfer and radiation shows a significant effect on the velocity as well as temperature distributions.

In analogy with the above works, in the present paper, the steady MHD free convection interaction with thermal radiation of an absorbing-emitting fluid along a vertical flat plate is investigated taking into account the Rosseland diffusion approximation. The investigation is based on known similarity analysis and the local similarity solutions are obtained numerically.

\section{Governing Equations}

Let us consider a steady two-dimensional MHD flow of a viscous, incompressible and electrically conducting fluid of temperature $T_{\infty}$ along a heated vertical flat plate under the influence of a uniform magnetic field. The flow is assumed to be in the $x$-direction, which is chosen along the plate in the upward direction and $y$-axis normal to the plate. The flow configuration and the coordinate system are shown in the Fig.1.

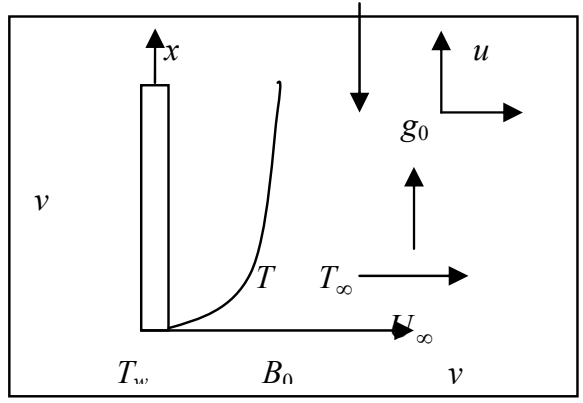

Fig. 1: Flow configuration and coordinate system.
The fluid is considered to be gray, absorbing emitting radiation but non-scattering medium and the Rosseland approximation is used to describe the radiative heat flux in the energy equation. The radiative heat flux in the $x$ direction is negligible to the flux in the $y$ direction. A uniform magnetic field of strength $B_{0}$ is applied normal to the plate parallel to $y$ direction. The plate temperature is initially raised to $T_{w}$ (where $T_{w}>T_{\infty}$ ) which is thereafter maintained constant.

Under the condition that the flow of viscous incompressible fluid in steady two-dimension, the boundary layer equations are

Continuity equation

$$
\frac{\partial u}{\partial x}+\frac{\partial v}{\partial y}=0
$$

and momentum equation

$$
u \frac{\partial u}{\partial x}+v \frac{\partial u}{\partial y}=v \frac{\partial^{2} u}{\partial y^{2}}
$$

which is treated by H. Blasius (1965), where $u$ and $v$ are the velocity components along $x$ and $y$ - directions respectively, $v$ is the kinematic viscosity. For free convection flow we have added the term $g_{0} \beta\left(T-T_{\infty}\right)$ where $\beta$ is the volumetric coefficient of thermal expansion, $g_{0}$ is the acceleration due to gravity, and $T \& T_{\infty}$ are the fluid temperature within the boundary layer and in the free-stream respectively. And the term $\frac{\sigma B_{0}{ }^{2} u}{\rho}$ stands for magnetic field where $\sigma$ is the electric conductivity, $\rho$ is the density of the fluid, $B_{0}$ is the uniform magnetic field strength (magnetic induction). Hence the momentum equation for the present problem takes the form

$u \frac{\partial u}{\partial x}+v \frac{\partial u}{\partial y}=v \frac{\partial^{2} u}{\partial y^{2}}+g_{0} \beta\left(T-T_{\infty}\right)-\frac{\sigma B_{0}^{2} u}{\rho}$

and the energy equation is given by

$u \frac{\partial T}{\partial x}+v \frac{\partial T}{\partial y}=\alpha \frac{\partial^{2} T}{\partial y^{2}}-\frac{1}{\rho c_{p}} \frac{\partial q_{r}}{\partial y}$

where $\alpha$ is the thermal diffusivity and the term $\frac{1}{\rho c_{p}} \frac{\partial q_{r}}{\partial y}$ introduces for radiation effect, $q_{r}$ is 
the radiative heat flux and $c_{p}$ is the specific heat at constant pressure.

Within the frame work of the above mentioned assumptions, we assume that the boundary layer and Boussinesq approximations hold and the flow and heat transfer in the presence of radiation are governed by the equations (1), (3) and (4).

By using Rosseland approximation (1976) $q_{r}$ takes the form

$q_{r}=-\frac{4 \sigma_{1}}{3 \kappa_{1}} \frac{\partial T^{4}}{\partial y}$

where, $\sigma_{1}$ is the Stefan-Boltzmann constant and $\kappa_{1}$ is the mean absorption coefficient. It is assumed that the temperature differences within the flow are sufficiently small such that $T^{4}$ may be expressed as a linear function of temperature. This is accomplished by expanding $T^{4}$ in a Taylor series about $T_{\infty}$ and neglecting higher-order terms, thus $T^{4} \cong T_{\infty}^{4}+\left(T-T_{\infty}\right) \cdot 4 T_{\infty}^{3}=4 T_{\infty}^{3} T-3 T_{\infty}^{4}$

Using equations (5) \& (6), eqn (4) takes the form

$u \frac{\partial T}{\partial x}+v \frac{\partial T}{\partial y}=\frac{\kappa}{\rho c_{p}} \frac{\partial^{2} T}{\partial y^{2}}+\frac{16 \sigma_{1} T_{\infty}^{3}}{3 \rho c_{p} \kappa_{1}} \frac{\partial^{2} T}{\partial y^{2}}$

where, $\kappa$ is the thermal conductivity.

Eventually the corresponding boundary conditions for the above problem are given by

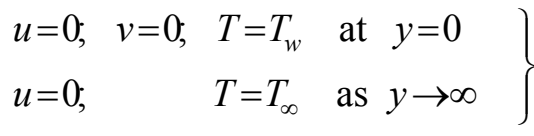

\section{Non-dimensionalization}

In order to obtain similarity solution of the problem the following non-dimensional variables are to be chosen

The suitable similarity variables, for the problem under consideration, are

$$
\begin{aligned}
& \eta=y \sqrt{\frac{U_{\infty}}{2 v x}}, \\
& \psi=\sqrt{2 v U_{\infty} x} f(\eta), \\
& \theta(\eta)=\frac{T-T_{\infty}}{T_{w}-T_{\infty}}
\end{aligned}
$$

where, $U_{\infty}$ is the free stream velocity .
Since $u=\frac{\partial \psi}{\partial y}$ and $v=-\frac{\partial \psi}{\partial x}$ we have the velocity components from eqn (8) given by

$$
\left.\begin{array}{l}
u=U_{\infty} f^{\prime}(\eta) \\
v=-\sqrt{\frac{v U_{\infty}}{2 x}}\left(\eta f^{\prime}-f\right)
\end{array}\right\}
$$

Here $f(\eta)$ is the dimensionless stream function and prime denotes the derivative with respect to $\eta$. Now introducing the similarity variables from eqn (9) and using eqn (10) into equation eqn (3) and (7) we have

$$
\begin{aligned}
& f^{\prime \prime \prime}+f f^{\prime \prime}+2 \frac{G r_{x}}{\operatorname{Re}_{x}^{2}} \theta-2 \frac{M}{\operatorname{Re}_{x}} f^{\prime}=0 \\
& (3 N+4) \theta^{\prime \prime}+3 N \operatorname{Pr} f \theta^{\prime}=0
\end{aligned}
$$

where

$G r_{x}=\frac{g \beta\left(T-T_{\infty}\right) x^{3}}{v^{2}}$ is the local Grashof number, $\operatorname{Re}_{x}=\frac{U_{\infty} x}{v}$ is the local Reynolds number, $M=\frac{\sigma B_{0}^{2} x^{2}}{\rho v}$ is the magnetic field parameter, $\operatorname{Pr}=\frac{v}{\alpha}=\frac{\rho v c_{p}}{\kappa}$ is the Prandtl number, $\quad N=\frac{\kappa \kappa_{1}}{4 \sigma_{1} T_{\infty}^{3}} \quad$ is the radiation parameter.

As a result the corresponding boundary conditions take the form

$$
\left.\begin{array}{r}
f=0, \quad f^{\prime}=0, \quad \theta=1 \quad \text { at } \eta=0 \\
f^{\prime}=0, \quad \theta=0 \quad \text { as } \eta \rightarrow \infty
\end{array}\right\}
$$

\section{Numerical Computation}

The numerical solutions of the nonlinear differential equations (11) \& (12) under the boundary conditions (13) have been performed by applying a shooting method namely Nachtsheim - Swigert (1965) iteration technique (guessing the missing value) along with sixth order Runge-Kutta integration scheme. We have chosen a step size of $\Delta \eta=$ 0.01 to satisfy the convergence criterion of $10^{-6}$ in all cases. The value of $\eta_{\infty}$ was found to each iteration loop by $\eta_{\infty}=\eta_{\infty}+\Delta \eta$. The maximum 
value of $\eta_{\infty}$ to each group of parameters $G r_{x}$, $\operatorname{Re}_{x}, M, \operatorname{Pr}$ and $N$ determined when the value of the unknown boundary conditions at $\eta=0$ not change to successful loop with error less than $10^{-6}$.

In order to verify the effects of the step size $(\Delta \eta)$ we ran the code for our model with three different step sizes as $\Delta \eta=0.01,0.005$ and 0.001 and in each case we found excellent agreement among them. Figure 2(a) \& 2(b) show the velocity and temperature profiles for different step sizes respectively.

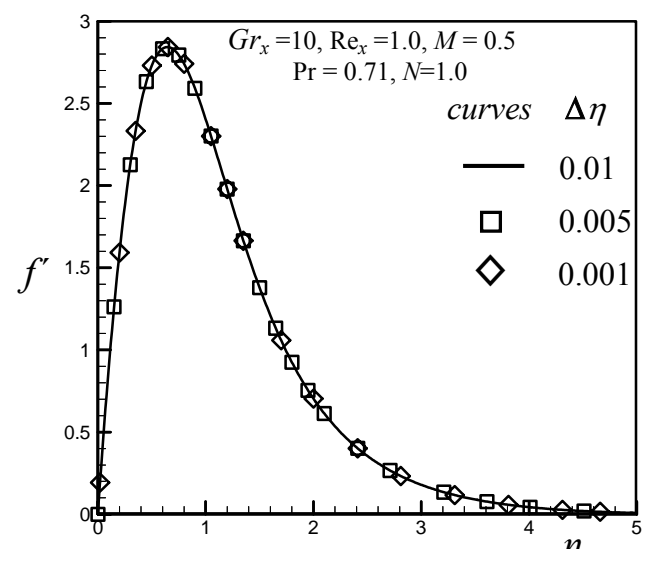

Fig. 2(a): Velocity profiles for different step size $\Delta \eta$.

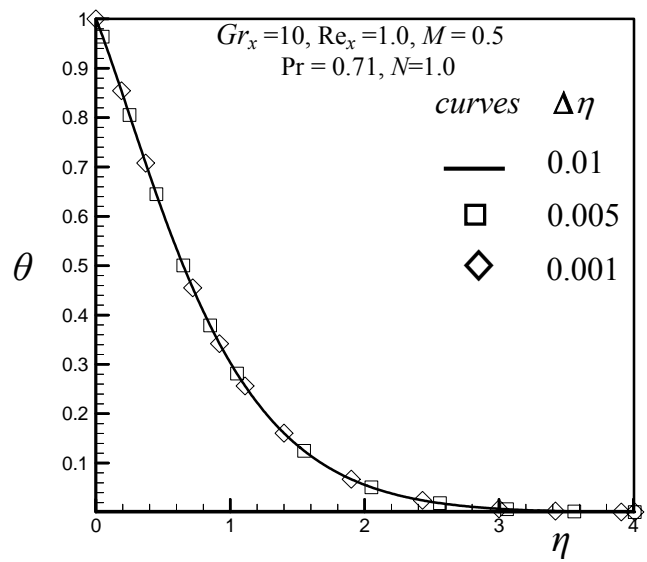

Fig. 2(b): Temperature profiles for different step size $\Delta \eta$.

\section{Results and Discussion}

In this chapter, the problem of the thermal radiation interaction with MHD boundary layer flow past a vertical flat plate in the presence of magnetic field has been investigated using Nachtsheim-Swigert (1965) shooting iteration technique. For the purpose of discussing the results, the numerical calculations are presented in the form of non-dimensional velocity and temperature profiles. Numerical computations have been carried out for different values of the Reynolds number $\operatorname{Re}_{X}$, Magnetic field parameter $M$, radiation parameter $N$, Prandtl number Pr and Grashof number $G r_{x}$. Since we consider a cooling problem only positive values of Grashof number are chosen.

Fig. 3(a) and 3(b) express the effect of Reynolds number $\left(\mathrm{Re}_{X}\right)$ on the velocity and temperature profiles respectively. From Fig 3(a) we see that the velocity decreases rapidly with the increase of $\mathrm{Re}_{x}$ but far away from the plate these profiles overlap whereas the temperature profiles increase monotonically with the increase of $\operatorname{Re}_{X}$ shown in Fig 3(b).

Fig 4(a) reveals the effect of magnetic field parameter $M$ on the velocity profiles. From this figure we see that velocity decreases with the increase of $M$ but far away from the plate these profiles overlap. As magnetic field increases the Lorentz force also increases which then tends to retard the motion of the fluid. Fig 4(b) depicts that temperature of the fluid increases as $M$ increases. Thus increasing the magnetic field intensity we can control flow and heat transfer mechanism.

Fig. 5(a) and Fig. 5(b) show the velocity and temperature profiles for different values of radiation number $N$. From Fig 5(a) we see that velocity decreases with the increase of the radiation parameter for cooling problem $\left(G r_{x}>\right.$ $0)$ as expected. We also see from Fig 5(b) that temperature decreases smoothly with the increase of $N$. So, one can control the flow characteristics using radiation.

The effect of Prandtl number Pr on the velocity as well as temperature profiles are shown in Fig. 6(a) $-6(\mathrm{~b})$. The velocity profile decreases with the increase of $\mathrm{Pr}$; this is due to the fact that as Pr increases the dynamic viscosity of the fluid increases which then slow down the 
velocity of the fluid. Fig. 6(b) shows that the Temperature decreases with the increase of Pr. In this study five different values are chosen where $\operatorname{Pr}=0.71$ corresponds to air at 200c and

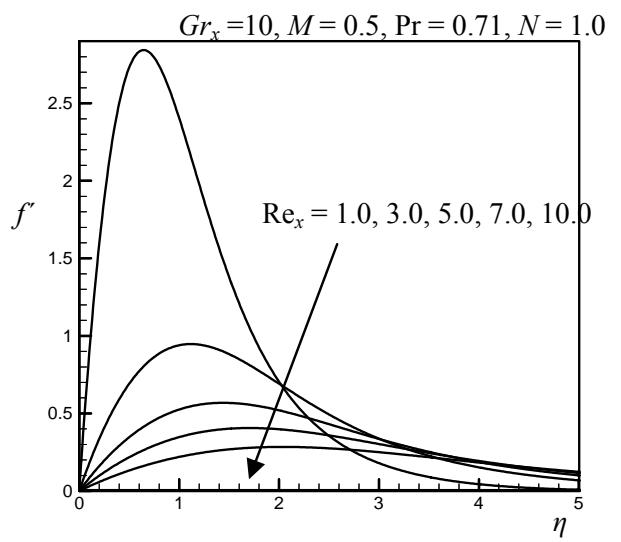

Fig. 3(a): Velocity profiles for various values of Reynolds number $\operatorname{Re}_{x}$.

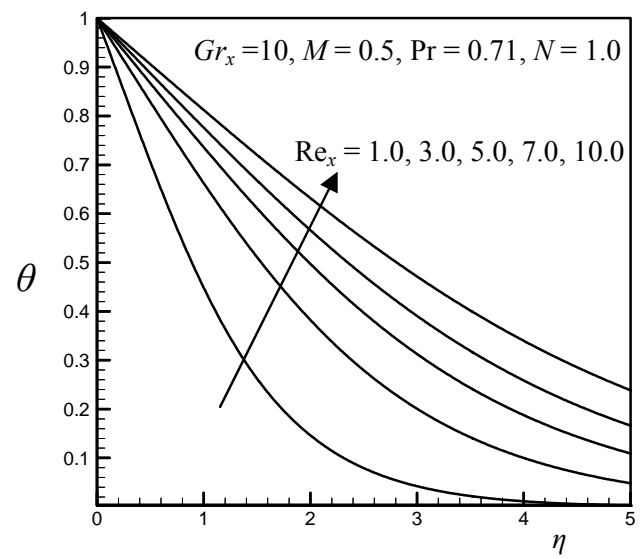

Fig. 3(b): Temperature profiles for various values of Reynolds number $\mathrm{Re}_{x}$.

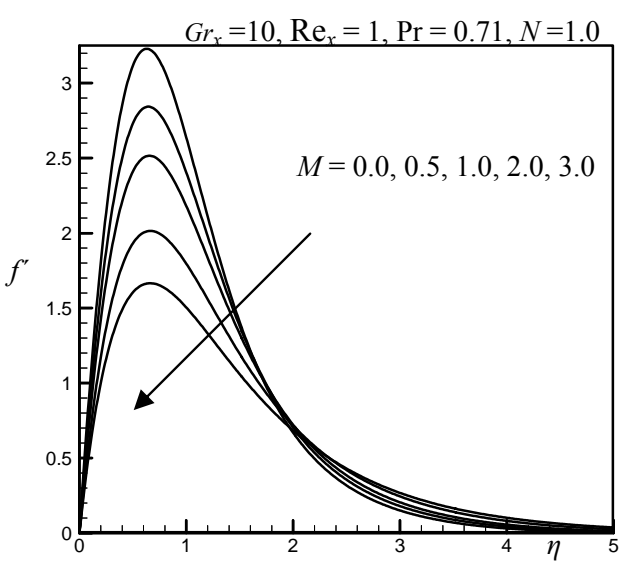

Fig. 4(a): Velocity profiles for various values of magnetic field parameter $M$.

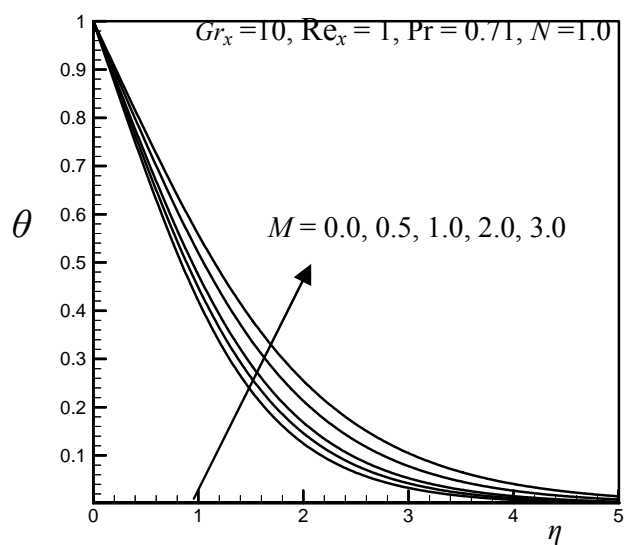

Fig.4(b): Temperature profiles for various values of magnetic field parameter $M$. 


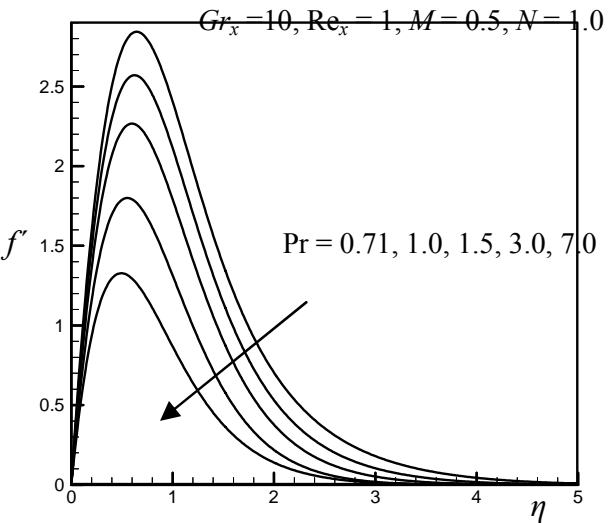

Fig.6(a): Velocity profiles for various values of Prandtl number Pr.

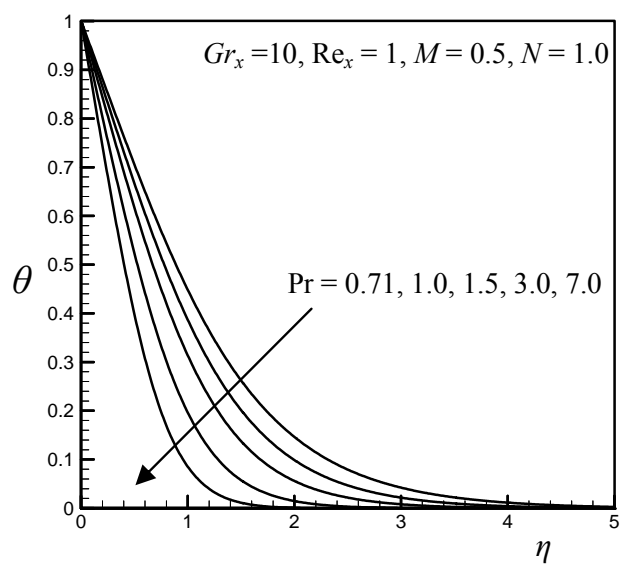

Fig. 6(b). Temperature profiles for various values of Prandtl number Pr.

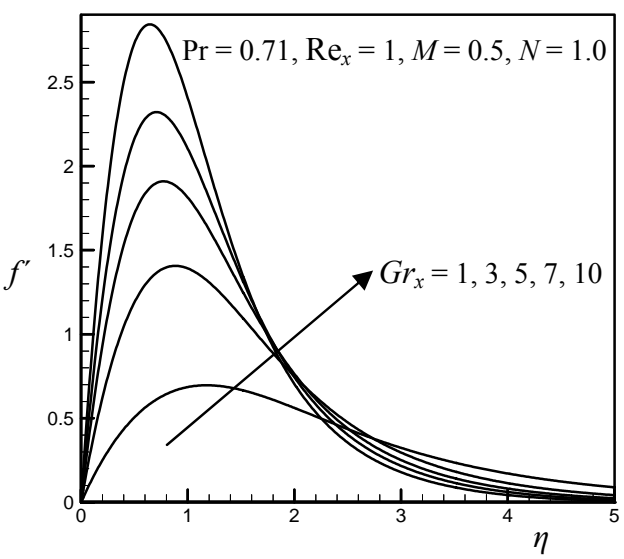

Fig.7(a): Velocity profiles for various values of Grashof number $G r_{x}$.

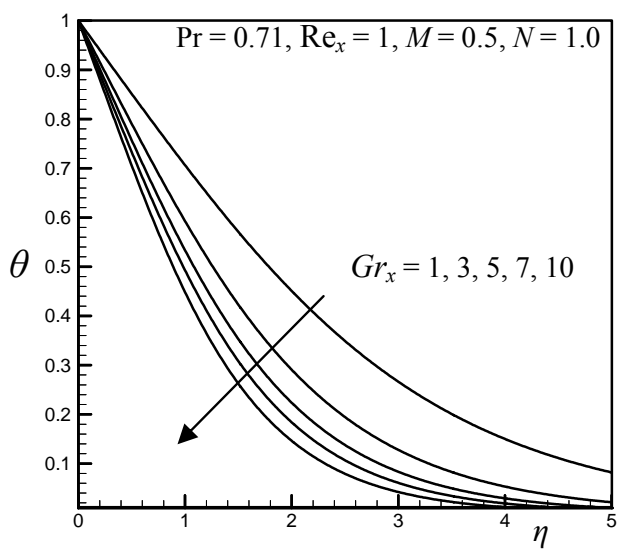

Fig.7(b): Temperature profiles for various values of Grashof number $G r_{x}$.

$\operatorname{Pr}=1.0$ corresponds to electrolyte solution such as salt water and $\operatorname{Pr}=7.0$ for water.

Fig. 7(a) indicates the velocity profiles showing the effect of the Grashof number $G r_{x}$. From this figure we see that velocity increases very rapidly as the increase of $G r_{x}$ due to the fact that as $G r_{x}$ increases, the buoyancy of the fluid increases which then induces the fluid velocity. Fig. 7(b) shows the temperature profiles for different values of $G r_{x}$. This figure reveals that temperature profiles decrease with the increase of $G r_{x}$. This is due to the fact that as $G r_{x}$ increases, the thermal state of the fluid close to the wall increases which then oppose the rate of heat transfer from the plate to the fluid.

The parameters of engineering interest for the present problem are the skin friction coefficient and local Nusselt number which indicate physically wall shear stress and rate of heat transfer respectively.

The skin-friction coefficient is given by

$c_{f}=\left(\frac{\mathrm{Re}_{x}}{2}\right)^{-\frac{1}{2}} f^{\prime \prime}(0)$

The local heat transfer coefficient is defined as

$$
N u_{x}=-\left(\frac{\operatorname{Re}_{x}}{2}\right)^{\frac{1}{2}} \theta^{\prime}(0)
$$

where $N u_{x}$ is the local Nusselt number. Thus from equations (14) \& (15) the values proportional to the skin-friction coefficient 
and Nusselt number are $f^{\prime \prime}(0)$ and $-\theta^{\prime}(0)$ respectively. The numerical values proportional to $c_{f}$ and $N u$, calculated from equations (14) \& (15) are shown in Table1.

Table 1. Values of skin friction $c_{f}$ and Nusselt number $N u_{x}$ for different values of $\operatorname{Re}_{x}, M, G r_{x}$, Pr and $N$.

$\begin{array}{ccc}\mathrm{Re}_{x} & c_{f} & N u_{x} \\ 1.0 & 9.84970320 & 0.59607034 \\ 3.0 & 1.89502879 & 0.34415683 \\ 5.0 & 0.88060164 & 0.26659201 \\ 7.0 & 0.53153146 & 0.22532093 \\ 10.0 & 0.31125023 & 0.18852744 \\ M & c_{f} & N u_{x} \\ 0.0 & 10.96468605 & 0.63209145 \\ 0.5 & 9.84970320 & 0.59607034 \\ 1.0 & 8.94715476 & 0.56331786 \\ 2.0 & 7.62087789 & 0.50771991 \\ 3.0 & 6.71642946 & 0.46368969 \\ G r_{x} & c_{f} & N u_{x} \\ 1 & 1.44229039 & 0.29779834 \\ 3 & 3.68454677 & 0.42098446 \\ 5 & 5.61984276 & 0.48949934 \\ 7 & 7.39061992 & 0.53914063 \\ 10 & 9.84970320 & 0.59607034 \\ \mathrm{Pr} & c_{f} & N u_{x} \\ 0.71 & 9.84970320 & 0.59607034 \\ 1.0 & 9.29733324 & 0.67720833 \\ 1.5 & 8.65996281 & 0.78306760 \\ 3.0 & 7.62295654 & 0.98991105 \\ 7.0 & 6.46555028 & 1.29183585 \\ N & c_{f} & N u_{x} \\ 1.0 & 9.84970320 & 0.59607034 \\ 2.0 & 9.30750130 & 0.67570691 \\ 3.0 & 9.08010636 & 0.71178225 \\ 5.0 & 8.87307619 & 0.74607763 \\ 7.0 & 8.77625675 & 0.76266776\end{array}$

\section{Conclusions}

In this paper we have studied the steady MHD free convection flow with thermal radiation in the presence of magnetic field past a vertical plate. Using similarity transformations the governing equations have been transformed into non-linear ordinary differential equations and were solved for similar solutions numerically.

From the present study we can make the following conclusions:

(i) The velocity profiles increase whereas temperature profiles decrease with an increase of the free convection current. (ii) Using magnetic field we can control the flow and heat transfer characteristics.

(iii) Radiation has significant effects on the velocity as well as temperature distribution.

(iv) Skin friction coefficient decreases with the increases of magnetic field parameter and radiation parameter.

(v) The rate of heat transfer from the plate to the fluid decreases with the increase of radiation parameter.

\section{References}

[1] R.M. Goody, "The influence of radiative transfer on cellular convection". Journal of Fluid Mechanics, Vol. 1, No. 04, 1956, pp. 424-435.

[2] R.D. Cess, "The Interaction of Thermal radiation with Free Convection Heat Transfer". Intl. J. Heat Mass Trans., Vol. 9, 1966, pp.1269-1277.

[3] J.L. Novotny, J.R. Lloyd and J.D. Bankston, AIAA Paper, Thermophysics and Heat Transfer Conference, Boston, Mass, USA, 1974, pp. 744-753.

[4] C.L. Tien and J.E. Lowder, "A correlation for total band absorptance of radiating gases". Int. J. Heat Mass Trans, Vol.9, No.7,1966,pp.698-701.

[5] V. M. Soundalgekar and H. S. Takhar, "MHD Flow Past a Vertical Oscillating plate". Nucl. Eng. Des., Vol.64, No.1, 1981, pp.43-48.

[6] M. M. Ali, T. S. Chen, and B.F. Armaly, "Natural Convection Radiation Interaction in Boundary Layer Flow over Horizontal Surfaces". AIAA Journal, Vol.22, No.12, 1984, pp.797-1803.

[7] M. A. Mansour, "Radiative and Free Convection Effects on the Oscillatory Flow Past a Vertical Plate". Astrophys. Space Sci., Vol.166, 1990, pp.269-275.

[8] M. A. Alabraba, A. R. Bestman and A. Ogulu, "Laminar Convection in Binary Mixed of Hydromagnetic Flow with Radiative Heat Transfer". Astrophys. Space Sci., Vol.195, 1992, pp. 431-445.

[9] G. Ahmadi "Self-similar solution of incompressible micropolar boundary layer flow over a semi-infinite plate”. Int. J. Eng. Sci., Vol.14, 1976, pp. 639.

[10] M.A. Sattar and H. Kalim, "Unsteady Free Convection Interaction with Thermal Radiation in a Boundary Layer Flow Past a Vertical Porous Plate". J. Math. Phys. Sci., Vol.30, No.1, 1996, pp.25-37.

[11] M. A. Hossain, H. S. Takhar, "Radiation Effect on Mixed Convection Along a Vertical Plate with Uniform Surface Temperature". Intl. J. Heat Mass Trans., Vol. 31, No.4, 1996, pp. 243-248.

[12] H. A. M. El-Arabawy, "Effect of Suction/Injection on a Micropolar Fluid Past a Continuously Moving Plate in the Presence of Radiation". Intl. J. Heat Mass Trans., Vol. 46, 2003, pp.1471-1477. 
[13] M. Ferdows, M.A. Sattar and M.N.A. Siddiki, "Numerical Approach on Parameters of the Thermal Radiation Interaction with Convection in a Boundary Layer Flow at a Vertical plate with Variable Suction". Thammmasat Intl J. Sci. Tech., Vol. 9, No.3, 2004, pp.19-28.

[14] M. A. Samad, and M.M. Rahman, "Thermal Radiation Interaction with Unsteady MHD Flow Past a Vertical Porous Plate Immersed in a Porous Medium". J. Nav. Arc. Mar. Eng., Vol.3, 2006, pp.7-14.
[15] P. R. Nachtsheim, and P. Swigert, "Satisfaction of Asymptotic Boundary Conditions in Numerical Solution of Systems of Non-linear Equations of Boundary Layer Type", NASA TN D-3004, 1965, PP. 1-37.

[16] H. Schlichting and K. Gersten, Boundary Layer Theory ( $8^{\text {th }}$ Revised and Enlarged Edition) Springer, 1987. 